\title{
Simon Claude Mimouni, Bernard Pouderon (dir.), La Croisée des chemins revisitée. Quand l'Église et la Synagogue se sont-elles distinguées?
}

Paris, Éditions du Cerf, coll. « Patrimoines. Judaïsme antique », 2012, $400 \mathrm{p}$.

Actes du colloque de Tours, 18-19 juin 2010

Anna van den Kerchove

\section{CpenEdition}

Journals

Édition électronique

URL : http://journals.openedition.org/assr/26502

DOI : $10.4000 /$ assr.26502

ISSN : $1777-5825$

Éditeur

Éditions de l'EHESS

Édition imprimée

Date de publication : 31 décembre 2014

Pagination : 251

ISBN : 978-2-7132-2467-6

ISSN : 0335-5985

Référence électronique

Anna van den Kerchove, «Simon Claude Mimouni, Bernard Pouderon (dir.), La Croisée des chemins revisitée. Quand l'Église et la Synagogue se sont-elles distinguées? », Archives de sciences sociales des religions [En ligne], 168 | 2014, mis en ligne le 11 mai 2015, consulté le 22 septembre 2020. URL http://journals.openedition.org/assr/26502 ; DOI : https://doi.org/10.4000/assr.26502

Ce document a été généré automatiquement le 22 septembre 2020.

(c) Archives de sciences sociales des religions 


\section{Simon Claude Mimouni, Bernard Pouderon (dir.), La Croisée des chemins revisitée. Quand l'Église et la Synagogue se sont-elles distinguées?}

Paris, Éditions du Cerf, coll. « Patrimoines. Judaïsme antique », 2012, $400 \mathrm{p}$.

Actes du colloque de Tours, 18-19 juin 2010

Anna van den Kerchove

\section{RÉFÉRENCE}

Simon Claude Mimouni, Bernard Pouderon (dir.), La Croisée des chemins revisitée. Quand l'Église et la Synagogue se sont-elles distinguées ? Paris, Éditions du Cerf, coll. « Patrimoines. Judaïsme antique », 2012, 400 p.

Actes du colloque de Tours, 18-19 juin 2010 
1 Le titre de l'ouvrage collectif dirigé par Simon Claude Mimouni et Bernard Pouderon, issu du colloque qui s'est tenu à Tours sur le même thème en 2012 est un écho à l'expression "The Parting of the Ways » que l'on doit essentiellement à James D. G. Dunn (lire en particulier The Parting of the Ways. Between Christianity and Judaism and Their Significance for the Character of Christianity, London, 1992, réédité en 2006) et qui a fait florès dans nombre d'articles et d'ouvrages; un écho qui, avec les termes «croisée » et " distinguer ", revisite cette expression et ce qu'elle implique. Il témoigne des débats de ces dernières années sur la manière d'envisager les rapports entre christianisme et judaïsme dans l'Antiquité, entre Église et Synagogue (pour reprendre les termes du sous-titre), et des renouvellements des hypothèses et des concepts. Quels termes employer?

Distinction, que préfère désormais Simon C. Mimouni (p.19)? Séparation, employé jusqu'à alors et qui continue à l'être par beaucoup (notamment dans cet ouvrage) ? Quand cela s'est-il produit? Au I ${ }^{\mathrm{er}}$ siècle, comme on l'a pensé longtemps? Au IV siècle, comme cela a été avancé plus récemment ? À l'heure actuelle, il semble qu'un certain consensus se fasse autour de l'hypothèse d'un processus qui a pu commencer dès le début $\mathrm{du} \mathrm{II}^{\mathrm{e}}$ siècle, mais qui est complexe et eut une périodisation longue. Enfin, quelles en sont les modalités? Les processus? Autant de questions qui sont à explorer, malgré les nombreux travaux qui existent déjà en la matière.

Simon C. Mimouni met l'ensemble de l'ouvrage en perspective avec un article introductif ("Introduction. Sur la question de la séparation entre "jumeaux" et "ennemis" aux I ${ }^{\text {er }}$ et $\mathrm{II}^{\mathrm{e}}$ siècles ", p. 7-20). Il interroge en particulier les concepts de " critique » et "vérité » (en se fondant sur Michel Foucault) et de "continuité » et "discontinuité ». C'est un moyen pour lui de rappeler ce que doit être l'histoire: critique, et donc démasquer les présupposés qui sont à l'œuvre et gênent, voire brouillent une analyse historique; et attachée à déceler les discontinuités, et pas seulement les continuités. Ce sont des rappels qu'il est toujours bon de faire, notamment pour ce qui concerne l'aspect critique, qui semble en effet être parfois oublié. Il revient aussi sur les derniers développements de la recherche sur le judaïsme antique - recherche à laquelle il participe lui-même -: on parle de plus en plus de judaïsme pluriel, et cette nouvelle façon de se représenter le judaïsme antique a des incidences sur la manière dont on analyse les rapports entre ce judaïsme et le christianisme. Enfin, il termine par le choix du «distinguer » au lieu de "séparer », dans l'idée de mettre en avant le cadre commun du christianisme et du rabbinisme.

3 Les quinze articles du volume (suivis chacun d'une bibliographie) traitent de la distinction, ou de la séparation, un terme que privilégient plusieurs auteurs. Que tous

Archives de sciences sociales des religions, 168 | 2014 
les auteurs n'adoptent pas la notion de «distinction » n'est en soi pas gênant, cela témoigne du débat en cours; en revanche, je trouve dommage qu'ils ne justifient pas le choix de cette notion, alors qu'elle est très présente dans la problématique même du colloque et de l'ouvrage. Plusieurs articles évoquent également, explicitement ou implicitement, les concepts de « discontinuité » et de « continuité ».

Pour les besoins de la recension, je proposerai un ordre différent de celui de l'ouvrage, qui, je l'espère, pourrait être utile au lecteur. Le premier article, de la plume d'Adriana Destre et de Mauro Pesce, "From Jesus Movement to Christianity: A Model for the Interpretation. Cohabitation and Separation of Jews and Christians » (p. 21-49). Ils reprennent les concepts de « discontinuités » et de " continuités », ainsi que le modèle heuristique de "système religieux » avec plusieurs composantes (p. 23). Ils utilisent également le concept de "cohabitation", en référence très probable au programme de recherche quadriennal (2006-2009) «Cohabitations et contacts religieux dans les mondes hellénistique et romain » (dir. Nicole Belayche et Jean-Daniel Dubois, EPHE) et auquel les auteurs de l'article ont participé. À partir de l'analyse des positions des disciples de Jésus, ils affirment que la discontinuité existe dès le départ. Ils en viennent ensuite, de manière intéressante, à l'étude de lieux précis: les tombes à Rome et les lieux d'habitation, pour montrer que des caractéristiques différentes apparaissent tôt, sans que Juifs et chrétiens ne constituent des groupes différents avant la fin du $\mathrm{II}^{\mathrm{e}}$ siècle.

5 Trois articles concernent l'Évangile selon Matthieu. Daniel Marguerat, dans «L'évangile de Matthieu et le judaïsme : un conflit de frères ennemis » (p. 51-64), analyse ce texte à l'aide de concepts issus de la sociologie (déviance, sectarisation) : l'évangile témoigne de débats internes au judaïsme, argumente contre le courant pharisien à l'aide de catégories internes au judaïsme, une situation qui ne se comprend que si on considère le judaïsme de l'époque comme étant sectarisé entre différents groupes opposés les uns aux autres. Edmondo Lupieri ("What Parting of Which Ways? The Gospel of Matthew as a Study Case», p. 107-124) s'interroge sur les «chemins». De quoi parle-t-on exactement ? Quel sens faut-il donner à ekklesia ? Lupieri répond à ces questions grâce à l'Évangile selon Matthieu qui construit, notamment à la fin de l'écrit avec le passage sur l'envoi en mission, son identité par rapport à des opposants juifs. Il conclut que cet écrit est le premier texte qui construit des "chemins", notion qui devient alors toute subjective. Le troisième article portant sur le texte matthéen aborde le texte selon une tout autre perspective : Elian Cuvillier, dans "Le paradigme du "déplacement" dans le processus de séparation entre l'évangile de Matthieu et le judaïsme du $\mathrm{I}^{\mathrm{er}}$ siècle " (p. 261-274), utilise le concept de « déplacement » qu'il définit ainsi : « un changement progressif de paradigme à l'intérieur d'un espace de croyance reconnu et assumé, au départ, comme légitime » (p. 263). À partir des thèmes de la Torah et du messianisme, du particularisme/universalisme et du langage parabolique, il étudie les écarts opérés par rapport au cadre commun. Cette notion d'écart, de déplacement s'ajoute à celle de discontinuité; elle me semble tout à fait intéressante et utile pour analyser les continuités et discontinuités.

Plusieurs articles élargissent le champ d'études, en abordant d'autres textes ou d'autres thèmes. Bernard Pouderon ( "Judaïsme et hérésie : étude sur les thèmes de l'exclusion chez les écrivains chrétiens $d u{ }^{\mathrm{e}}$ siècle ", p. 65-89) s'attache, de manière très intéressante, à étudier comment les protagonistes de l'époque ont perçu la réalité, ce qui modifie la manière de lire et analyser les écrits de l'époque, qu'ils soient Juifs, chrétiens (dont les gnostiques) ou païens. Relevons que pour les Juifs, à côté de sources 
talmudiques (dont la datation fait débat), B. Pouderon utilise Tryphon : on a alors, me semble-t-il, plus le regard d'un chrétien (Justin) sur la manière dont les Juifs pouvaient percevoir la réalité. Par ailleurs, cela montre le faible nombre de mentions des chrétiens et de Jésus dans les écrits juifs de l'époque, ce qui en soi est intéressant. Il conclut que pour les Juifs, les chrétiens sont des déviants, ce que les intéressés ne pensent pas être ; les gnostiques, à quelques exceptions près, distinguent les chrétiens des Juifs, même s'ils les assimilent pour les besoins de la polémique ; Juifs et chrétiens ne sont pas encore "séparés » au $\mathrm{II}^{\mathrm{e}}$ siècle. Il évoque les judéo-chrétiens, dont il estime que c'est peut-être un "faux problème » à cette époque (p. 86). Avec "Once Again Birkat Hammimim Revisited» (p. 91-105), Daniel Boyarin complète l'article de Bernard Pouderon, en réexaminant un texte juif bien connu. Dans ces pages, il répond à des critiques qui lui ont été adressées. Il reprend donc le dossier de ce texte avec les témoignages anciens sur une imprécation contre les chrétiens. Il conclut en réaffirmant l'hypothèse d'un « parting of the ways » tardif.

7 Pierluigi Piovanelli, dans « De l'usage polémique des récits de la Passion, ou Là où les chemins qui auraient dû se séparer ont fini par se superposer » (p. 125-160), met sur le devant de la scène de ce débat les écrits apocryphes : le Livre du coq, l'Évangile de Judas et Toledoth Yeshu, qui offrent différentes descriptions (et interprétations) de Jésus, ainsi que d'autres écrits, comme l'Évangile du Sauveur, le Discours du Sauveur à propos de la croix et la Danse du Sauveur autour de la croix. Les différences narratives et les positions variées des auteurs concernant les Juifs ou les acteurs principaux (Jésus, Judas, etc.) témoignent, selon Piovanelli, de situations socioculturelles variées. L'auteur affirme également, à l'encontre d'opinions actuelles, que les positions doctrinales des gnostiques relèvent moins d'une prise de position antijuive que d'une mise en perspective autre de l'héritage juif (p. 151), «ayant pris définitivement leur distance par rapport aux Juifs ». Comme il le dit, cela incite à réévaluer ce que disent les gnostiques par rapport aux autres chrétiens et Juifs. Robert Michael Edwards, dans "Jewish Christianity Revisited: The Jewish Christian Background of the First Apocalypse of James (NHC V, 3; AMC 2)» (p.321-337), revient sur un texte souvent négligé : la Première Apocalypse de Jacques, connue sous deux versions (celle découverte à Nag Hammadi et celle du codex Tchacos). Après une analyse des deux versions, il se concentre sur la manière dont l'autorité de Jacques est fondée. Selon R. M. E., cet écrit témoigne que les lignes entre christianisme et judaïsme sont encore floues dans certaines contrées de l'Empire romain à la fin del'Antiquité.

Claire Clivaz ( Jacob and Jesus in Alexandria as a Test-Case: "The Eldest Angel” and "The Mystic Angel”", p. 213-225) s'intéresse à la présence ou l'absence (en soi significative) de motifs comme celui de Jacob. Annette Yoshiko Reed («Parting Ways over Blood and Water? », p. 227-260) commence par des remarques heuristiques sur les limites (voire les dangers) de la microhistoire qui ont un risque de myopie (p. 234). Il ne faudrait pas oublier, selon elle, que des concepts créés dans un contexte particulier ont connu ensuite une grande fortune et ont contribué à formuler une vision d'une réalité au-delà d'une époque et d'un lieu donnés. Elle donne l'exemple du ioudaismos d'Ignace. On pourrait également évoquer la catégorie eusébienne de " reste » dont il est question dans l'article de Sébastien Morlet. Elle en vient au propos de son article : le sang, l'eau et la pureté et la manière dont ils sont évoqués dans des écrits juifs et chrétiens dans le cadre de constructions identitaires. Cet article pourrait être complété par celui de José Costa qui propose, avec « Le marqueur identitaire de la circoncision chez les rabbins de 
l'Antiquité »(p.161-194), une analyse fine d'écrits juifs relatifs à la circoncision, montrant l'évolution de la position des rabbins.

Gilles Dorival, dans «La Bible, un canon partagé ou des canons séparés?» (p. 195-212), analyse les différents regroupements de textes bibliques dont il est question dans les sources juives et chrétiennes et des expressions qui servent à dénommer ces regroupements. L'auteur parle ainsi d'une évolution, avec un canon partagé, jusqu'aux environs de 200, puis un canon séparé.

Enfin, plusieurs études concernent plus particulièrement le $\mathrm{IV}^{\mathrm{e}}$ siècle, moment où des chercheurs ont voulu placer la séparation. Sébastien Morlet («Le "reste d'Israël” selon Eusèbe de Césarée: théologie, exégèse et histoire d'une catégorie frontalière ", p. 275-319) étudie la façon dont la première histoire de l'Église évoque Israël et le judaïsme ; il s'intéresse en particulier à la catégorie « eusébienne » de reste (reprise de $\mathrm{Rm}$ 11.5). Il montre ainsi qu'Eusèbe ne conçoit pas la séparation comme ayant une histoire, mais comme faisant partie de l'identité chrétienne dès le départ. Une idée qui a des influences importantes sur la manière dont les chrétiens (et les chercheurs) ont jusqu'à récemment perçu la séparation d'avec le judaïsme. Alain Le Boulluec revient sur l'accusation de "judaïser» qui a été portée contre des adversaires chrétiens, par d'autres chrétiens: "Arius judaizans? Crise de mutation et signe de séparation?» (p. 299-319). Il analyse les ressorts de la polémique, en particulier l'amalgame (même si cette notion développée dans son La Notion d'hérésie dans la littérature grecque [II ${ }^{e}-I I I^{e}$ siècles], Paris, Études augustiniennes, 1985, est ici absente). Il montre comment cette accusation conduit à substituer aux vrais Juifs les " hérétiques » et les "Juifs de jadis ", ce qui contribue, à côté de l'influence de l'histoire eusébienne, à renforcer l'idée d'une distinction originelle. Cet article complète ainsi l'article de Simon Mimouni. Dominique Côté ( Le problème de l'identité religieuse dans la Syrie du IV siècle. Le cas des PseudoClémentines et de l'Adversus Judaeos de saint Jean Chrysostome », p. 339-370). L'auteur y reprend la notion d'habitus de Bourdieu, afin d'évoquer la situation à Antioche, caractérisée par le pluralisme religieux et par des leaders cherchant à imposer à une société plurielle les règles d'une communauté particulière.

11 Nombre d'articles sont de qualité et aident à mieux cerner les termes du débat, en particulier du point de vue méthodologique et heuristique. Plusieurs auteurs proposent des concepts ou des outils d'analyse intéressants pour la recherche ultérieure. Cet ouvrage fournit ainsi une nouvelle pierre pour une meilleure compréhension d'un phénomène complexe, celui de la distinction plus ou moins progressive entre Juifs et chrétiens, entre judaïsme et christianisme. 